\title{
List of abbreviations
}

AEF American Expeditionary Force

AFS American Field Service

ANC Army Nurse Corps

ARC American Red Cross

BEF British Expeditionary Force

BGH British General Hospital

CBC Canadian Broadcasting Company

CIA Central Intelligence Agency

CPT Captain

DMZ demilitarized zone

DSM Diagnostic and Statistical Manual of Mental Disorders

ESWS Ex-Services' Welfare Society

GI US private soldier

IED improvised explosive device

LMF lack of moral fibre

MASCAL mass casualty

MEDCAP Medical Civil Action Program

MO medical officer

POW prisoner of war

PTSD post-traumatic stress disorder

QAIMNS members of Queen Alexandra's Imperial Military

Nursing Service

RAF Royal Air Force

RAMC Royal Army Medical Corps

RMO regimental medical officer

SJAB St John's Ambulance Brigade

USAID US government aid agency 
List of abbreviations

USMC US Marine Corps

VA Veterans Association

VAD Voluntary Aid Detachment

VVA Vietnam Veterans Association 